\title{
b-continuity and Partial Grundy Coloring of graphs with large girth *
}

\author{
Allen Ibiapina and Ana Silva \\ ParGO Group - Paralelism, Graphs and Optimization \\ Departamento de Matemática \\ Universidade Federal do Ceará \\ Fortaleza, CE - Brazil \\ allenr.roossimegmail.com, anasilva@mat.ufc.br
}

\begin{abstract}
A b-coloring of a graph is a proper coloring such that each color class has at least one vertex which is adjacent to each other color class. The b-spectrum of $G$ is the set $S_{b}(G)$ of integers $k$ such that $G$ has a b-coloring with $k$ colors and $b(G)=\max S_{b}(G)$ is the b-chromatic number of $G$. A graph is bcontinous if $S_{b}(G)=[\chi(G), b(G)] \cap \mathbb{Z}$. An infinite number of graphs that are not b-continuous is known. It is also known that graphs with girth at least 10 are b-continuous. A partial Grundy coloring is a proper coloring $f: V(G) \rightarrow$ $\{1, \ldots, k\}$ such that each color class $i$ contains some vertex $u$ that is adjacent to every color class $j$ such that $j<i$. The partial Grundy number of $G$ is the maximum value $\partial \Gamma(G)$ for which $G$ has a partial Grundy coloring.

In this work, we prove that graphs with girth at least 8 are b-continuous, and that the b-spectrum of a graph $G$ with girth at least 7 contains the integers between $2 \chi(G)$ and $b(G)$. We also prove that $\partial \Gamma(G)$ equals a known upper bound when $G$ is a graph with girth at least 7 . These results generalize previous ones by LinharesSales and Silva (2017), and by Shi et al.(2005).
\end{abstract}

\section{Introduction}

Let $G$ be a simple graph (for basic terminology on graph theory, we refer the reader to [4]). A function $\psi: V(G) \rightarrow \mathbb{N}$ is a proper $k$-coloring of $G$ if $|\psi(V(G))|=k$ and $\psi(u) \neq \psi(v)$ whenever $u v \in E(G)$. Because we only deal with proper colorings in this text, from call them simply a coloring. We call the elements of $\psi(V(G))$ colors. Given a color $i \in \psi(V(G))$, the set $\psi^{-1}(i)$ is called color class $i$. The chromatic number of $G$ is the minimum value $\chi(G)$ for which $G$ admits a coloring. The problem of deciding $\chi(G) \leq k$ for given $G$ and $k$ is one of the most studied problems in Graph Theory. It is one of Karp's 21 NP-complete problems [12], and it remains NP-complete even when restricted to many classes of graphs, as for instance, it is NP-complete when $k=3$ and $G$ is a 4-regular planar graph [7], or a line graph [10]. There are many variations of the coloring problem, and in this article we investigate two of these variations, both of them based on existing coloring heuristics.

\footnotetext{
* Partially supported by CNPq Projects Universal no. 401519/2016-3 and Produtividade no. 304576/2017-4, and by FUNCAP/CNPq project PRONEM no. PNE-0112-00061.01.00/16.
} 
Given a coloring $\psi$ of $G$, we say that $u \in V(G)$ is a $b$-vertex in $\psi$ if $u$ is adjacent to every color class different from $\psi(u)$, i.e., if $N(u) \cap \psi^{-1}(i) \neq \emptyset$ for every $i \neq \psi(u)$. Note that if some color class $c$ does not contain b-vertices, we can obtain a $(k-1)$ coloring by changing the color of each vertex $v \in \psi^{-1}(c)$ to another color different from $c$ where $v$ has no neighbors. We say that this new coloring is obtained from the first one by cleaning color $c$. If a coloring cannot be cleaned, it means that all color classes have at least one b-vertex. Such a coloring is called a $b$-coloring of $G$. Observe that an optimal coloring cannot have the number of colors decreased by the described algorithm; therefore every optimal coloring is also a b-coloring. In [11], the authors define the $b$-chromatic number of $G$, denoted by $b(G)$, as the largest natural $k$ for which $G$ has a b-coloring with $k$ colors. In the same article, the authors demonstrated that the problem of finding $b(G)$ is NP-complete in general.

Another interesting aspect about b-colorings concerns its existence for every possible value between $\chi(G)$ and $b(G)$. In [11], the authors observe that the cube has a b-coloring using 2 colors and 4 colors, but has no b-coloring using 3 colors. Inspired by this result, in [13] it is shown that for any integer $n \geq 4$ the graph obtained from the complete bipartite graph $K_{n, n}$ by deleting the edges from a perfect matching has a bcoloring using 2 and $n$ colors, but has no b-coloring using a number of colors between 2 and $n$. This motivates the definition of the $b$-spectrum of $G$, that is the set $S_{b}(G)$ containing every integer $k$ such that $G$ has a b-coloring with $k$ colors. A graph $G$ is $b$-continous if $S_{b}(G)=[\chi(G), b(G)] \cap \mathbb{Z}$. In [3], they prove that for each finite subset $S \subset \mathbb{N}-\{1\}$, there exists a graph $G$ such that $S_{b}(G)=S$, and also that deciding if a graph is b-continuous is NP-complete even if colorings with $\chi(G)$ and $b(G)$ colors are given.

Now, given a b-coloring with $k$ colors, since each b-vertex has at least $k-1$ neighbors, there exists $k$ vertices with degree at least $k-1$ (this would be a subset of $k$ b-vertices of the $k$ colors). So if we define $m(G)$ as the largest positive integer $k$ such that there exist at least $k$ vertices with degree at least $m(G)-1$ in $G$, we have that $b(G) \leq m(G)$. This upper bound was introduced in [11], where the authors show that one can find $m(G)$ in polynomial time using the degree list of the graph. Also, they prove that if $G$ is a tree, then $b(G) \geq m(G)-1$, and that one can decide if $b(G)=m(G)$ in polynomial time. Their result was later generalized for graphs with girth at least 7 [20] (the girth of $G$ is the minimum lenght of a cycle in $G$ ). We also mention that there are many results that say that regular graphs with large girth have high b-chromatic number [17|5[18 17]. Indeed, the following conjecture is still open.

Conjecture 1 (Blidia, Maffray and Zemir [17]). If $G$ is a $d$-regular graph with girth at least 5 and $G$ is not the Petersen graph, then $b(G)=d+1$.

Because of these results, it makes sense to investigate the b-continuity of graphs with large girth. Indeed, in [1] the authors prove that regular graphs with girth at least 6 and without cycles of length 7 are b-continuous, and in [15], they prove that every graph with girth at least 10 are b-continuous. Here, we improve their result to graphs with girth at least 8 .

Theorem 1. If $G$ is a graph with girth at least 8 , then $G$ is b-continuous. 
In addition, we prove that graphs with girth at least 7 are, in a way, almost bcontinuous.

Theorem 2. If $G$ is a graph with girth at least 7 , then $[2 \chi(G), b(G)] \cap \mathbb{Z} \subseteq S_{b}(G)$.

Now, given a coloring $\psi: V(G) \rightarrow\{1, \ldots, k\}$, we say that $u \in V(G)$ is a Grundy vertex if $u$ has a neighbor in color class $i$, for every color $i<f(u)$, and that $f$ is a partial Grundy coloring if every color class contains at least one Grundy vertex. This concept was introduced in [8], where the authors relate the new concept with parsimonious colorings. We mention that a more largely studied concept is that of the Grundy colorings, where every vertex is a Grundy vertex. These are also called greedy colorings since they represent colorings that can be obtained by the greedy algorithm. In this context, a partial Grundy coloring can be thought of as a coloring that cannot have the number of colors decreased by moving vertices to smaller colors. Because a coloring with $\chi(G)$ colors cannot be improved like this, we get that it must be a partial Grundy coloring. The partial Grundy number is then defined as the worst case scenario for this heuristis, i.e., it is the maximum number of colors in any partial Grundy coloring; it is denoted by $\partial \Gamma(G)$.

Interestingly enough, these colorings are also easier on graphs with large girth. In [19], the authors introduce an upper bound for $\partial \Gamma(G)$, called the stair factor of $G$ and denoted here by $s(G)$, and they prove that if $G$ has girth at least 9, then $\partial \Gamma(G)=s(G)$. We improve their result in the theorem below.

Theorem 3. If $G$ is a graph with girth at least 7 , then $\partial \Gamma(G)=s(G)$, and an optimal partial Grundy coloring can be obtained in polynomial time.

Observe that this and the result in [20] tell us that that if $G$ has girth at least 7, then both the b-chromatic number and the partial Grundy number of $G$ are high. However, we mention that these colorings are not similar when the continuity is concerned. In [2], the authors prove that every graph $G$ has a partial Grundy coloring with $k$ colors, for every $k \in\{\chi(G), \ldots, \partial \Gamma(G)\}$. This is a common trait with Grundy colorings [6].

Our article is organized as follows. In Section 2 , we prove Theorems 11 and 2 , in Section 3, we prove Theorem 3, and in Section 4, we give our closing remarks. The definitions and notation are introduced as they are needed.

\section{2 b-continuity}

In [1], a vertex $u \in V(G)$ is called a $k$-iris if there exists $S \subseteq N(u)$ such that $|S| \geq$ $k-1$ and $d(v) \geq k-1$ for every $v \in S$. See Figure 1. This definition is important because of the following important lemma.

Lemma 1 ([1]). Let $G$ be a graph with girth at least 6 and without cycles of lengh 7. If $G$ has a $k$-iris with $k \geq \chi(G)$, then $G$ has a b-coloring with $k$ colors.

To prove Theorems 1 and 2 given a b-coloring of $G$ with $k$ colors, $k>\chi(G)+1$, we try to obtain a b-coloring of $G$ with $k-1$ colors. However, this is not always possible, and when this happens, it is because we have a $k$-iris. Our theorem then follows from 


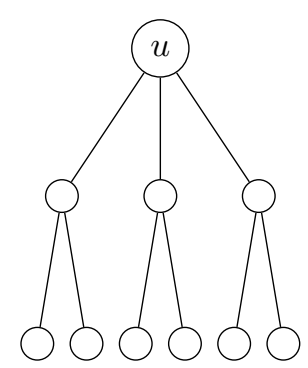

Fig. 1. In the figure, we present a 4-iris.

the lemma above. We mention that the constraint about not having cycles of length 7 appears only in the above lemma, but not on our proof. We now introduce the further needed definitions.

Let $G$ be a simple graph and $\psi$ be a b-coloring of $G$ with $k>\chi(G)+1$ colors. We say that $u$ realizes color $i$ if $\psi(u)=i$ and $u$ is a b-vertex. We also say that color $i$ is realized by $u$. For $x \in V(G)$ and $i \in\{1, \ldots, k\}$, let $N^{\psi, i}(x)$ be the set of vertices of color $i$ in the neighborhood of $x$, i.e., $N^{\psi, i}(x)=N(x) \cap \psi^{-1}(i)$; in fact, we omit $\psi$ in the superscript since it is always clear from the context. This is also done in the next definitions. For a subset $X \subseteq V(G)$, let $N^{i}(X)=\bigcup_{x \in X} N^{i}(X) \backslash X$. Let $B(\psi)$ denote the set of b-vertices in $\psi$ and, for each $i \in\{1, \ldots, k\}$, let $B_{i}=B(\psi) \cap \psi^{-1}(i)$ be the set of b-vertices in color class $i$.

Given a set $K$ such that $K \subseteq \psi^{-1}(i)$ for some $i \in\{1, \ldots, k\}$, we say that a color $j \in\{1, \ldots, k\} \backslash\{i\}$ is dependent on $K$ if $N^{i}\left(B_{j}\right) \subseteq K$; denote by $U(K)$ the set of colors depending on $K$. If $K=\{x\}$, we write simply $U(x)$. Given $x \in V(G) \backslash B(\psi)$, if $|U(x)| \geq 2$ we call $x$ a useful vertex; otherwise, we say that $x$ is useless. For $j \in$ $\{1, \ldots, k\}$, we say that $x \in V(G)$ is $j$-mutable if $x$ is useless and there exists a color $c$ such that we can change the color of $x$ to $c$ without creating any b-vertex of color $j$; we also say that color $c$ is safe for $(x, j)$. If there is no safe color for $(x, j)$, we say that $x$ is $j$-imutable.

The next lemma is the main ingredient in our proof. Combined with Lemma 1, it immediatly implies Theorem 1

Lemma 2. Let $G=(V, E)$ be a graph with girth at least 7. If $G$ has a b-coloring with $k$ colors where $k \geq \chi(G)+1$, then either $G$ has a b-coloring with $k-1$ colors, or $G$ contains a $(k-1)$-iris.

Proof. Our proof is similar to that made in [15], but we concentrate in one color that we want to eliminate.

Suppose that $G$ does not have a b-coloring with $k-1$ colors; we prove that $G$ has a $(k-1)$-iris. For this, let $\psi$ be a b-coloring with $k$ colors that minimizes $\left|B_{1}\right|$ and then minimizes $\left|\psi^{-1}(1)\right|$ (i.e., it firstly minimizes the number of $b$-vertices of color 1 , then it minimizes the number of vertices of color 1). First, we prove that every $x \in$ $\psi^{-1}(1) \backslash B_{1}$ is useful. Suppose otherwise and let $x$ be a useless vertex in color class 1 , 
i.e., $|U(x)| \leq 1$. If $U(x)=\emptyset$, then we can recolor $x$ without losing any b-vertex, a contradiction since $\psi$ minimizes $\left|\psi^{-1}(1)\right|$. And if $U(x)=\{d\}$, then we can obtain a b-coloring with $k-1$ by recoloring $x$ and cleaning $d$, again a contradiction. Therefore, the following holds:

(i) Every $x \in \psi^{-1}(1) \backslash B_{1}$ is useful.

Now, we choose any $u \in B_{1}$ and analyse its vicinity in order to obtain the desired $(k-1)$-iris. For this, the following two claims are essential.

Claim. Let $j \in\{2, \ldots, k\}$. If every $x \in N^{j}(u) \backslash B_{j}$ is 1-mutable, then one of the following holds:

(ii) $N(u) \cap B_{j} \neq \emptyset$; or

(iii) There exists a color $d \in\{2, \ldots, k\} \backslash\{j\}$ such that $d$ depends on $N^{j}(u)$, i.e.,

$$
N^{j}\left(B_{d}\right) \subseteq N^{j}(u) .
$$

Proof of claim: Suppose that neither (ii) nor (iii) holds, and let $\psi^{\prime}$ be obtained from $\psi$ by changing the color of each $x \in N^{j}(u)$ to a color $c$ safe for $(x, 1)$. Because (iii) does not hold, we get that $U\left(N^{j}(u)\right) \subseteq\{1\}$. Therefore, at most one color loses all of its b-vertices, namely color 1 , and since every $x \in N^{j}(u)$ is 1-mutable, no b-vertices of color 1 is created. But because $u$ is not a b-vertex in $\psi^{\prime}$ (it is not adjacent to color $j$ anymore) and $\psi$ minimizes $\left|B_{1}\right|$, we get that $\psi^{\prime}$ cannot be a b-coloring, which means that we can obtain a b-coloring with $k-1$ colors by cleaning color $1 . \diamond$

The following claim tells us that (ii) or (iii) actually always hold.

Claim. (iv) Every $x \in N^{j}(u) \backslash B_{j}$ is 1-mutable, for every $j \in\{2, \ldots, k\}$.

Proof of claim: Suppose, without loss of generality, that $d \in\{2, \ldots, k\}$ is such that the colors in $\{d+1, \ldots, k\}$ are exactly the colors that contains some 1 -imutable vertex. We count the number of colors with b-vertices in the vicinity of $u$ to get that in fact $d \geq k$. So, for each $i \in\{d+1, \ldots, k\}$, let $w_{i} \in N^{i}(u)$ be a 1 -imutable vertex. By definition, this means that, for each $i \in\{d+1, \ldots, k\}$, there exists some neighbor of $w_{i}$ that would be turned into a b-vertex of color 1 in case we change the color of $w_{i}$; let $v_{i}$ be such a vertex. We then know that $v_{i} \in \psi^{-1}(1) \backslash B_{1}$, which by (i) gets us that $\left|U\left(v_{i}\right)\right| \geq 2$. By the definition of $U(x)$ and the fact that every $x \in\left\{v_{d+1}, \ldots, v_{k}\right\}$ is colored with color 1 , we get:

$$
U\left(v_{i}\right) \cap U\left(v_{\ell}\right)=\emptyset \text {, for every } i, \ell \in\{d+1, \ldots, k\}, i \neq \ell .
$$

Now, we investigate the b-vertices around colors $\{2, \ldots, d\}$. By Claim 2 suppose, without loss of generality, that $p \in\{2, \ldots, d\}$ is such that (ii) holds for colors in $\{2, \ldots, p\}$, while (iii) holds for colors in $\{p+1, \ldots, d\}$. For each $i \in\{p+1, \ldots, d\}$, let $c_{i} \in\{2, \ldots, k\} \backslash\{i\}$ be a color depending on $N^{i}(u)$, which means that $B_{c_{i}} \subseteq$ $N\left(N^{i}(u)\right)$. Observe that, since $G$ has no cycles of length 3, we get:

$$
\{2, \ldots, p\} \cap\left\{c_{p+1}, \ldots, c_{d}\right\}=\emptyset
$$


Also, because $G$ has no cycles of length 4 , we get $c_{i} \neq c_{\ell}$ for every $i \neq \ell$, i.e.:

$$
\left|\left\{c_{p+1}, \ldots, c_{d}\right\}\right|=d-p
$$

Finally, because $G$ has no cycles of length smaller than 6 , we get that:

$$
\left\{2, \ldots, p, c_{p+1}, \ldots, c_{d}\right\} \cap \bigcup_{i=d+1}^{k} U\left(v_{i}\right)=\emptyset .
$$

Now, recall that $\psi\left(v_{i}\right)=1$ for every $i \in\{d+1, \ldots, k\}$, and that $c_{i} \neq 1$ for every $i \in\{p+1, \ldots, d\}$. This means that $1 \notin\left\{c_{p+1}, \ldots, c_{d}\right\} \cup \bigcup_{i=d+1}^{p} U\left(v_{i}\right)$. By combining Equations (1) through (4), we get the following, which implies $d \geq k$ as desired:

$$
\begin{aligned}
k-1 & \geq\left|\{2, \ldots, p\} \cup\left\{c_{p+1}, \ldots, c_{d}\right\} \cup U\left(v_{d+1}\right) \cup \ldots \cup U\left(v_{k}\right)\right| \\
& =d-1+\sum_{i=d+1}^{k}\left|U\left(v_{i}\right)\right| \\
& \geq d-1+2(k-d) .
\end{aligned}
$$

Now, let $N=(N(u) \cup N(N(u))) \backslash\{u\}$. Observe that because (ii) or (iii) holds for every color $\ell \in\{2, \ldots, k\}$, we get that $B(\psi) \subseteq N$. Suppose that $N[u]$ does not contain a $(k-1)$-iris, otherwise the proof is done. This means that at least one color in $\{2, \ldots, k\}$, say $k$, is such that (ii) does not hold for $k$, which by Claim 2 implies that (iii) holds, i.e., that there exists a color in $\{2, \ldots, k-1\}$, say 2 , such that $N^{2}\left(B_{k}\right) \subseteq N^{2}(u)$ (Observe Figure 2). Now, let $w \in N^{1}\left(B_{k}\right)$; it exists since the vertices in $B_{k}$ are bvertices. By (i), there exists at least two colors in $\{2, \ldots, k\}$ that depend on $w$. But because $B(\psi) \subseteq N$, we get a cycle of length at most 6 , a contradiction.

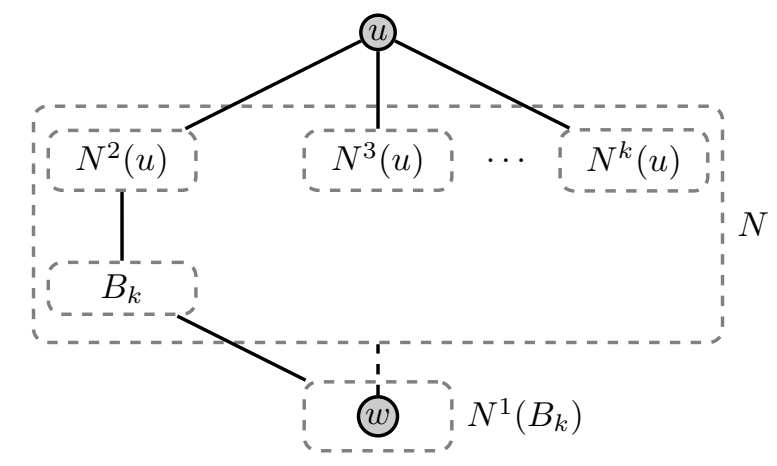

Fig. 2. Structure around $u$ when color $k$ does not satisfy Claim 2(ii).

Now, to prove Theorem 2, we apply Lemma 2 and the next lemma. A star is a tree that has at most one vertex with degree bigger than 1, and the diameter of a graph $G$ is the maximum number of edges in a shortest path of $G$. 
Lemma 3. If a graph $G$ has girth at least 7 and a $k$-iris where $k \geq 2 \chi(G)$, then $G$ has a b-coloring with $k$ colors.

Proof. Let $u \in V(G)$ be a $k$-iris with $k \geq 2 \chi(G)$. Let $u_{2}, \ldots, u_{k}$ be neighbors of $u$ such that $d\left(u_{i}\right) \geq k-1$ for every $i \in\{2, \ldots, k\}$; let $N_{i}$ be a subset of $k-2$ neighbors of $u_{i}$ different from $u$. Start by coloring $u$ with 1 and, for each $i \in\{2, \ldots, k\}$, give color $i$ to $u_{i}$ and colors $\{2, \ldots, k\} \backslash\{i\}$ to $N_{i}$. Denote by $P$ the set $\bigcup_{i=2}^{k} N\left[u_{i}\right]$ (set of colored vertices), and observe that the coloring can be easily done since $G[T]-u$ is a forest formed by $k-1$ stars. Also, note that we already have $k$ b-vertices of distinct colors, and thus it only remains to extend the partial coloring to the rest of the graph. For this, let $B$ be the set of vertices adjacent to some color class in $\{1, \ldots, \chi(G)\}$. See Figure 3 .

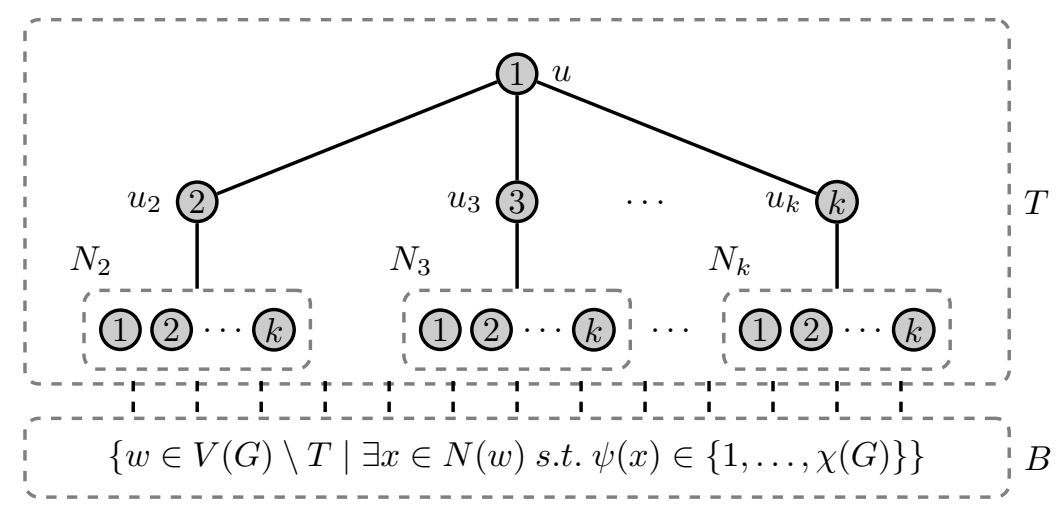

Fig. 3. Subset of vertices around $u$. The label inside a vertex denotes its color.

We claim that $|N(w) \cap T| \leq 1$ for every $w \in B$; indeed, because $T$ induces a tree of diameter 4 , if this was not true than we would get a cycle of length at most 6 . By the definition of $B$, we then get that every $w \in B$ has no neighbors in color classes $\chi(G)+1, \ldots, k$. Thus, since $k \geq 2 \chi(G)$, we can color $G[B]$ with colors $\chi(G)+$ $1, \ldots, 2 \chi(G)$. Finally, by the definition of $B$, we know that every $w \in V(G) \backslash(B \cup T)$ has no neighbors of color 1 through $\chi(G)$, which means that we can color $G-T-B$ with these colors.

\section{Partial Grundy Colorings}

The next definitions were introduced in [19]. Given $W=\left(w_{1}, \ldots, w_{s}\right)$, a sequence of vertices, we say that $W$ is a feasible sequence if $d_{G_{i}}\left(w_{i}\right) \geq i-1$, for every $i \in$ $\{1, \ldots, s\}$, where $G_{i}=G-\left\{w_{i+1}, \ldots, w_{s}\right\}$. The stair factor of $G$ is the maximum size of a feasible sequence; we denote it here by $s(G)$. In [19], they prove that $s(G)$ is an upper bound for $\partial \Gamma(G)$, and that if $W$ is a feasible sequence of $G$ of size $s$, and $G$ 
has girth at least 9, then $G$ has a partial Grundy coloring with $s$ colors. This implies that $\partial \Gamma(G)=s(G)$ when $G$ has girth at least 9 . We prove that it continues to hold when $G$ has girth at least 7 by also proving that the existence of a feasible sequence of size $s$ makes it possible to obtain a partial Grundy coloring of $G$ of size $s$. We mention that they also prove that computing a feasible sequence of maximum size can be done in polynomial time, which combined with our proof imply Theorem 3 .

Now, consider a feasible sequence $\left(w_{1}, \ldots, w_{s}\right), s \geq 2$, and for each $i \in\{2, \ldots, s\}$, let $N_{i} \subseteq N_{G_{i}}\left(w_{i}\right)$ be of cardinality $i-1$. Denote the set $\left\{w_{2}, \ldots, w_{s}\right\}$ by $W$. We say that a partial coloring $\psi$ of $G$ is non-redundantfor $W$ if for every $i \in\{2, \ldots, s\}$, we get that $\psi\left(w_{i}\right)=i$ and all the colored vertices in $N_{i}$ have distinct colors in $\{1, \ldots, i-1\}$.

Proof (of Theorem 3). Let $\left(w_{1}, \ldots, w_{s}\right)$ be a feasible sequence. Denote by $W$ the set $\left\{w_{2}, \ldots, w_{s}\right\}$ and let $N_{i}$ be defined as before; also let $N=\bigcup_{i=2}^{s} N_{i}$. First, note that if $\psi$ is a non-redundant partial coloring that colors $W \cup N$, then a partial Grundy coloring with at least $s$ colors can be obtained from $\psi$ in a greedy way: this is true because $w_{i} \in$ $W$ is a Grundy vertex in $\psi^{-1}(i)$, for each $i \in\{2, \ldots, s\}$, and any vertex $x \in \psi^{-1}(1)$, which exists since $N_{2}$ is colored with 1 , is a Grundy vertex.

Start by coloring each $w_{i} \in W$ with $i$. For each $x \in N$, define:

$$
m(x)=\min \left\{i \in\{2, \ldots, s\} \mid w_{i} \in N(x)\right\} .
$$

Note that if $\psi$ is a non-redundant partial coloring and $x$ is colored in $\psi$, then $\psi(x)<$ $m(x)$. We will then color $N$ in a non-decreasing order of $m(x)$. Suppose we are at the iteration of some $x \in N$, and observe that the colors that are forbidden for $x$ are the colors that appear in $N(x)$, and also the colors that appear in $N\left(w_{i}\right)$, where $w_{i} \in N(x)$. So, denote by $\ell$ the value $m(x)$ and by $F$ the set of vertices $N(x) \cup N\left(N_{W}(x)\right)$. By what was said before, if there exists a color $c \in\{1, \ldots, \ell-1\} \backslash \psi(F)$, then we can give color $c$ to $x$ to obtain a non-redundant partial coloring that colors $x$, so suppose otherwise. For each $i \in\{1, \ldots, \ell-1\}$, let $y_{i} \in F$ be such that $\psi\left(y_{i}\right)=i$. First, note that, by the definition of $m(x)$ and the fact that $\psi\left(y_{i}\right)<m(x)$, we get that if $y_{i} \in N(x)$, then $y_{i} \in N$. Now, for each $i \in\{1, \ldots, \ell-1\}$, define:

$$
u_{i}= \begin{cases}y_{i}, & \text { if } y_{i} \in W \\ w_{m\left(y_{i}\right)}, & \text { otherwise }\end{cases}
$$

Let $W^{<\ell}$ be the set $\left\{w_{2}, \ldots, w_{\ell-1}\right\}$. Observe Figure 4 .

Consider $i \in\{1, \ldots, \ell-1\}$. If $u_{i}=y_{i}$, then, because $\psi\left(y_{i}\right)<m(x)$, we get $u_{i} \in$ $W^{<\ell}$. Otherwise, we get that $y_{i} \in N$ and, by the chosen order of coloring, we get that $m\left(y_{i}\right)<m(x)$, which again implies that $u_{i} \in W^{<\ell}$. We then get $\left\{u_{1}, \ldots, u_{\ell-1}\right\} \subseteq$ $W^{<\ell}=\left\{w_{2}, \ldots, w_{\ell-1}\right\}$, which by the pigeonhole principle implies that there exist $i, j \in\{1, \ldots, \ell-1\}$ with $i \neq j$ such that $u_{i}=u_{j}$. But because $u_{i}$ and $u_{j}$ are at distance at most 3 from $x$, we get a cycle of length smaller than 7 , a contradiction.

\section{Conclusion}

We have proved that every graph with girth at least 8 is b-continuous, and that graphs with girth at least 7 are in a way almost b-continuous. This improves the result presented 


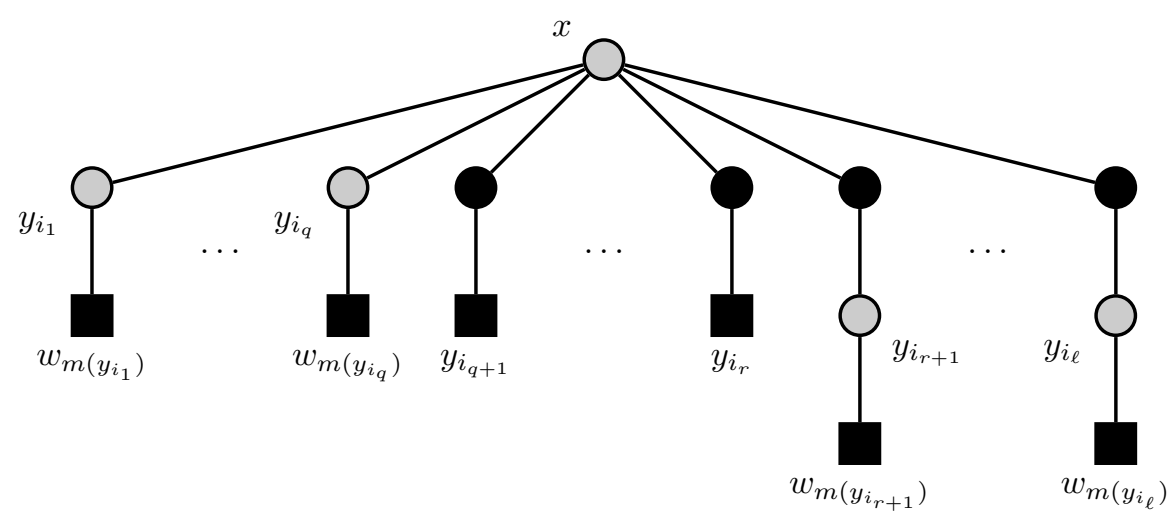

Fig. 4. Subset of vertices in the vicinity of $x$. Black vertices are in $W$. Square vertices are in $\left\{u_{1}, \ldots, u_{\ell}\right\}$. Observe that no two of them are equal since $G$ has girth at least 7 .

in [15], where they prove that graphs with girth at least 10 are b-continuous. There, the authors also pose the following questions:

Question 1. What is the minimum $\hat{g}$ such that $G$ is b-continuous whenever $G$ is a graph with girth at least $\hat{g}$ ?

Question 2. What is the minimum $\tilde{g}$ such that $G$ is b-continuous whenever $G$ is a bipartite graph with girth at least $\tilde{g}$ ?

Recall that the graph obtained from the complete bipartite graph $K_{n, n}$ by removing a perfect matching is not b-continuous, for every $n \geq 4$ [13]. Hence, by our result we get:

$$
5 \leq \hat{g} \leq 8
$$

And because bipartite graphs have no odd cycles, we get:

$$
\tilde{g} \in\{6,8\} .
$$

We believe that the same techniques might improve this bound to 7 , but not further. In particular, we mention that Lemma 2 works for graphs with girth 7 , and that Theorem 1 is restricted to girth at least 8 just because of Lemma 1 . Therefore, if the following question is answered "yes", then we get $\hat{g} \leq 7$.

Question 3. Let $G$ be a graph with girth at least 7 such that $G$ has a $k$-iris, with $k \geq$ $\chi(G)+1$. Does $G$ admit a b-coloring with $k$ colors?

We also mention that the coloring problem is NP-complete for graphs with girth at least $k$, for every fixed $k \geq 3$ [16]. This is why any proof of a result like Theorem 1 is expected to have a non-constructive part. 
As for the case of bipartite graphs, we think it is worth mentioning a known conjecture about their b-chromatic number. Recall the upper bound $m(G)$ for the b-chromatic number $b(G)$, which is the maximum value $k$ for which there exist $k$ vertices with degree at least $k-1$. The set of all vertices with degree at least $m(G)-1$ is denoted by $D(G)$, and a graph is said to be tight if $|D(G)|=m(G)$; this means that there is only one candidate set for the set of b-vertices of a b-coloring of $G$ with $m(G)$ colors. Deciding if $b(G)=m(G)$ is NP-complete even for bipartite tight graphs [13]. In [9], the authors define the class $\mathcal{B}_{m}$ that contains every bipartite graph $G=(A \cup B, E)$ such that $m(G)=m, D(G)=A$ and $G$ has girth at least 6 . They conjecture the following:

Conjecture 2 (Havet, Linhares-Sales and Sampaio [9]). For every $m \geq 3$, and every $G \in \mathcal{B}_{m}$, we have that:

$$
b(G) \geq m(G)-1
$$

We mention that, if $G$ is a bipartite graph with girth at least 6 and a b-coloring of $G$ with $k$ colors is given, with $k \geq \chi(G)+1$, then, with a little more work in the proof of Lemma 2, one can get that $G$ contains an induced subgraph $H$ that has a structure similar to the structure of a graph in $\mathcal{B}_{k}$. Trying to use this structure to obtain a bcoloring of $H$ with $k-1$ colors could translate into proving Conjecture 2 . And on the other way around, we believe that a strategy to prove Conjecture 2 could help coloring these graphs, which would imply that the answer to Question 2 is "yes". This means that answering Question 2 seems as hard as proving Conjecture 2 We also mention that in [14], it is proved that Conjecture 2 is a consequence of the famous Erdôs-FaberLovász Conjecture, which remains open since 1972 and which is largely believed to hold. This is strong evidence that Conjecture 2 holds.

Now, concerning partial Grundy colorings, an obvious question also concerns the minimum girth for which $\partial \Gamma(G)=s(G)$ holds.

Question 4. What is the minimum $\dot{g}$ such that $\partial \Gamma(G)=s(G)$ whenever $G$ is a graph with girth at least $\dot{g}$ ?

Oberve that if $G=(A \cup B, E)$ is the complete bipartite graph $K_{n, n}$, then $s(G)=$ $n+1$ : a feasible sequence is obtained by starting with any vertex in $A$, then ordering each $v \in B$. However, we argument that the only existing partial Grundy coloring of $G$ uses 2 colors. For this, suppose without loss of generality that color 1 appears in $A$. Then no vertex in $B$ is colored with 1 , which means that no Grundy vertex of color bigger than 1 can appear in $A$. But now, if color $i>2$ has a Grundy vertex $u$ in $B$, then color 2 must appear in $A$ and, therefore, cannot appear in $B$. We get a contradiction since in this case color 2 has no Grundy vertices. We then get the following bounds:

$$
5 \leq \dot{g} \leq 7
$$

\section{References}

1. R. Balakrishnan and T. Kavaskar. b-coloring of kneser graphs. Discrete Appl. Math., 160:9$14,2012$. 
2. R. Balakrishnan and T. Kavaskar. Interpolation theorem for partial grundy coloring. Discrete Math., 313(8):949-950, 2013.

3. D. Barth, J. Cohen, and T. Faik. On the b-continuity property of graphs. Discrete Appl. Math., 155:1761-1768, 2007.

4. A. Bondy and U.S.R. Murty. Graph Theory. Spring-Verlag Press, 2008.

5. S. Cabello and M. Jakovac. On the b-chromatic number of regular graphs. Discrete Appl. Math., 159:1303-1310, 2011.

6. C.A. Christen and S.M. Selkow. Some perfect coloring properties of graphs. Journal of Combinatorial Theory B, 27:49-59, 1979.

7. D.P. Dailey. Uniqueness of colorability and colorability of planar 4-regular graphs are npcomplete. Discrete Math., 30(3):289-293, 1980.

8. P. Erdôs, S.T. Hedetniemi, R. Laskar, and G.C.E. Prins. On the equality of the partial grundy and upper ochromatic numbers of graphs. Discrete Math., 272(1):53-64, 2003.

9. F. Havet, C. Linhares-Sales, and L. Sampaio. b-coloring of tight graphs. Discrete Appl. Math., 160(18):2709-2715, 2012.

10. I. Holyer. The np-completeness of edge-coloring. SIAM J. on Computing, 10(4):718-720, 1981.

11. R.W. Irving and D.F. Manlove. The b-chromatic number of a graph. Discrete Appl. Math., 91:127-141, 1999.

12. R. Karp. Reducibility among combinatorial problems. Complexity of Computations, Advances in Computer Research, 85-103, 1972.

13. J. Kratochvíl, Zs. Tuza, and M. Voigt. On the b-chromatic number of graphs. In WG 2002 Int. Workshop on Graph-Theoretic Concepts in Comp. Sc., 2002.

14. W.-H. Lin and G.J. Chang. b-coloring of tight bipartite graphs and the erdos-faber-lovász conjecture. Discrete Appl. Math., 161(7-8):1060-1066, 2013.

15. C. Linhares-Sales and A. Silva. The b-continuity of graphs with large girth. Graphs and Combinatorics, 33(5):1139-1146, 2017.

16. V.V. Lozin and M. Kaminski. Coloring edges and vertices of graphs without short or long cycles. Contributions do Discrete Mathematics, 2(1), 2007.

17. F. Maffray M. Blidia and Z. Zemir. On b-colorings in regular graphs. Discrete Appl. Math., 157:1787-1793, 2009.

18. A. El Sahili and H. Kouider. About b-colouring of regular graphs. Utilitas Math., 80:211$215,2009$.

19. Z. Shi, W. Goddard, S.T. Hedetniemi, K. Kennedy, R. Laskar, and A. McRae. An algorithm for partial Grundy number on trees. Discrete Math., 304:108-116, 2005.

20. C. Lima V. Campos and A. Silva. Graphs with girth at least 7 have high b-chromatic number. European Journal of Combinatorics, 48:154-164, 2015. 